\title{
Etude du comportement de l'homme en milieu souterrain
}

(Bilan de cinq experimentations)

Par Pierre Saumande ${ }^{1}$ )

Asec planches 14 (1)-15 (2)

Le milieu souterrain, celui des grottes et des gouffres parait particulièrement hostile à l'homme : obscurité totale profonde, humidité importante, température relativement basse, niveau sonore bas ou très spécial (réverbération des sons sur les parois, rivières souterraines) paraissant autant de facteurs impropres à une vie normale.

La spéléologie a prouvé, par des expéditions de longue durée, que l'on pouvait séjourner sous terre dans les cavités naturelles.

Il nous a paru intéressant de connaître comment l'homme s'y comportait.

Nos recherches entreprises au début dans un but uniquement pratique (amélioration du rendement, prévention des accidents) sont devenues plus théoriques étant donné les résultats obtenus.

\section{Methodes et techniques}

Dans l'établissement du protocole expérimental que nous devions concevoir, nous avions le choix entre deux solutions :

- effectuer les tests et examens en surface avant l'entrée dans la cavité et les renouveler à la sortie

- ou, au contraire, contrôler les sujets régulièrement, en continu, en les accompagnant pendant leur séjour souterrain.

En nous basant sur nos observations, lors d'expéditions spéléologiques courantes, nous avons opté pour la deuxième solution.

Cette décision nous obligeait à étudier tout particulièrement les examens que nous devions pratiquer. Il les fallait simples, faciles à réaliser, ne nécessitant qu'un matériel léger, résistant, tout en permettant d'obtenir des résultats rigoureux.

1) Ecole Nationale de Médecine et de Pharmacie Limoges (France). 
Il fallait aussi que les tests soient bien acceptés par les sujets volontaires auxquels nous allions faire appel, ce qui excluait les examens sanguins.

Une recherche bibliographique nous ayant montré qu'il n'y avait que peu de travaux publiés dans ce domaine, (l'analyse de ceux-ci a été faite dans la communication présentée au Congrès de Bordeaux intitulée "Physiologie en Spéléologie») e'est aux examens utilisés dans l'étude des rations alimentaires spéciales pour milieux d'exception, que nous avons arrêté notre choix.

Le Bideau 1961 - Genneseaux 1956 - Medical Nutrition Laboratory 1956 - Metz, Lambert, Hasselman, van Hove 1959 - Placidi, Postic 1955 - Rivolier 1955.

Les épreuves biologiques et les tests de contrôle

Lors de la première expérience (Saumande - Le Bideau 1962) que nous considérions comme "expérience pilote», nous avons effectué :

- une mesure de la pression artérielle au lever.

- un test de Ruffier-Dickson modifié par Garetta.

(C'est un test d'aptitude générale à l'effort. Le sujet effectue 30 flexions sur les jambes en 45 secondes, selon une fréquence régulière d'une flexion toutes les secondes et demie. Une minute exactement après la fin des flexions, on compte le pouls pendant 15 secondes. Le tableau établi par Garetta permet de classer les sujets.)

- (Sur les urines récoltées en totalité, chaque sujet est muni d'un flacon de 2 litres en matière plastique et d'un flacon plastique de $750 \mathrm{~cm} .^{3}$ pour les déplacements.)

- la mesure du volume.

- la détermination de la densité.

- la détermination du $\mathrm{pH}$.

- la recherche de l'albumine, de l'acétone, du glucose.

- un dosage de potassium et du sodium.

- un dosage de l'urée.

- un dosage de l'azote total.

L'examen des résultats nous ayant montré que la mesure de la pression artérielle ne fournissait aucune indication permettant de tirer une conclusion, nous avons supprimé cette mesure par la suite.

Il en a été de même pour le test de Ruffier-Dickson. Devant la difficulté à établir le bilan azoté, nous avons décidé de supprimer la détermination de l'azote total. C'est donc avec cette série réduite d'examens que nous avons continué nos recherches lors des expériences suivantes. 
Nous y avons ajouté, lors de la deuxième (Saumande 1964) un dosage de l'ammoniaque urinaire. On connaît depuis très longtemps la rapport existant entre l'élimination des ions $\mathrm{H}^{+}$et le débit ammoniacal du rein ; l'étude du $\mathrm{pH}$ et de l'ammoniaque nous a montré une corrélation à peu près exacte.

Enfin, tout au long de nos différentes expériences nous avons dosé les mucoprotéines urinaires (Tayeau 1960).

\section{Examens complémentaires}

Nous avons soumis les sujets à un test psycho-physiologique, «test d'estimation des durées brèves», tel qu'il est décrit par Angiboust. L'intérêt de cette épreuve n'est pas de rechercher avec quel degré de précision un individu donné estime la seconde. L'intervalle d'une seconde a été choisi parce qu'il exprime une donnée intuitive facilement appréhensive par tous. Des études expérimentales et cliniques ont montré que pour un individu donné la longueur de son estimation variait en raison inverse de l'état d'éveil général de son organisme. Plus les estimations sont brèves, plus l'état d'éveil est élevé et vice versa. L'intérêt de cette épreuve est de pouvoir chiffrer l'intensité de ces états d'éveil (Angiboust 1962).

Nous avons aussi procédé, lors des quatrième et cinquième expériences, à la détermination de la température rectale.

Enfin, nous avons ajouté aux examens prévus pour la cinquième expérience, une étude de l'acuité visuelle, un test de Farnsworth 100 Hue au début et à la fin du séjour souterrain (Perdriel 1962) et un examen hématologique.

(Pour les techniques utilisées quant à la mesure de l'humidité et de la température, je renvoie à la communication faite au Congrès de Bordeaux : «Etude de quelques facteurs physiques pouvant avoir une influence sur le comportement de l'homme séjournant dans les cavités naturelles.")

\section{Les conditions de l'expérience}

\section{L'environnement}

Toutes nos expériences ont eu lieu dans des cavités de grandes dimensions permettant l'installation d'un camp de base confortable, l'installation d'un petit laboratoire et présentant suffisamment de ressources du point de vue spéléologique pour intéresser les participants.

Les premières expériences out eu lieu dans des cavités de basse altitude dont la température moyenne était de 10 à $12^{\circ} \mathrm{C}$ et l'hygrométrie 
Speleology III. Saumande

de $100 \%$. La température des rivières qui les parcouraient variait de 10 à $14^{\circ} \mathrm{C}$ (rivière souterraine du Saut, rivière souterraine de la Reille [Dordogne], rivière souterraine de Padirac, rivière souterraine des Vitarelles [Lot]). Pour la dernière de nos expériences nous avons choisi une cavité beaucoup plus froide $: 4^{\circ}$, parcourue par un torrent dont l'eau était aussi à $4^{\circ}$ (Gouffre de la Pierre St-Martin [BassesPyrénées]).

\section{Les sujets}

Nous avons toujours fait appel à des volontaires jeunes (de 17 à 30 ans), sportifs entrainés, en bonne forme physique. Tous ont été préalablement avertis des conditions générales de l'expérience.

\section{Les conditions de vie}

Les sujets étaient logés dans des tentes canadiennes d'un modèle classique, avec double toit et tapis de sol. Tous les participants étaient dotés d'un matelas pneumatique et d'un sac de couchage en duvet.

L'alimentation était constituée, en grande partie, de conserves et d'aliments concentrés. La valeur énergétique moyenne de la ration alimentaire était de 3.500 calories par jour. Cette ration était répartie en 3 prises, de la façon suivante :

- au début de la journée, un petit déjeuner avec café au lait sucré ou «Ovomaltine», pain, margarine et confiture.

- vers le milieu de la journée, une ration condensée d'exploration : biscuits, chocolat, charcuterie, fruits secs et aliments spéciaux, aliment «Montblanc» ou tablettes d' "Ovomaltine» destinés à être absorbés en cours de déplacement.

- en fin de journée, un repas cuisiné comportant : bouillon concentré viande ou poisson, légumes ou pâtes, fromage, confiture.

Tous les jours, les sujets, répartis en équipes, ont eu un programme d'exploration spéléologique à réaliser. Nous pensons, en effet, en nous référant au comportement des équipiers chargés d'assurer pendant de longues heures des relais à l'entrée de puits souterrains, qu'il était impérieux pour les sujets en expérience, de mener une vie active, l'inaction dans les grottes produisant chez l'homme un effet de somnolence.

Nous nous sommes efforcés aussi d'avoir en expérience un nombre assez grand de personnes, un résultat isolé étant difficile à interpréter. Nous avons pu soumettre à nos tests des groupes comportant respectivement $5,9,15,17$ et 21 sujets. 
Les séjours souterrains ont eu des durées variables de 4 à 8 jours permettant ainsi de contrôler efficacement l'action du milieu, sans pour cela lasser les sujets et réduire la collaboration que nous attendions d'eux.

Malgré le séjour de groupes assez importants dans les cavités, les résultats des mesures de température et d'humidité ont été les mêmes au début et à la fin de l'expérience, les dimensions des cavités et leur ventilation étant très grandes.

\section{Les résultats}

\section{Résultats et discussion}

Pour ne pas alourdir cet exposé par des tableaux de résultats numériques, nous nous sommes contentés de donner en annexe leur transcription en courbes qui expriment les données en valeurs moyennes pour l'ensemble des groupes.

La pression artérielle ne fournit aucune indication permettant de tirer une conclusion. Nous n'avons pas constaté le «pincement de la différentielle» signalé par Dufour.

Le test de Ruffier-Dickson montre une légère amélioration du coefficient. Il semble que l'épreuve ait eu chez tous les sujets, malgré les difficultés, la valeur d'un entraînement aux efforts intenses.

En ce qui concerne les urines, nous avons noté quelques discrètes et rares protéinuries de fatigue, une progression du $\mathrm{pH}$, de l'acidité à la neutralité.

L'étude du pH et de l'ammoniaque des urines nous a montré une corrélation à peu près exacte.

L'importance de l'acidose pouvant être en rapport avec la fatigue des sujets, nous avons pensé qu'il serait peut-être possible d'avoir une idée de leur état physique en dosant l'ammoniaque urinaire, la détermination du $\mathrm{pH}$ sanguin ne pouvant être effectuée dans nos conditions expérimentales.

Le volume urinaire, malgré une légère pointe la $3^{\circ}$ ou $4^{\circ}$ journée du séjour souterrain, décroît régulièrement. Cette diminution peut être attribuée à une absorption réduite de liquide (les sujets, n'ayant à leur disposition que de l'eau froide $10^{\circ}$ et $4^{\circ}$, ne boivent guère plus que les quelques liquides chauds prévus dans la ration alimentaire).

- La densité urinaire suit dans le temps une évolutions grossièrement inverse de celle du volume urinaire.

- L'urée urinaire qui présente une très forte diminution au début du séjour, continue à décroître mais beaucoup plus lentement. 
- L'excrétion du sodium suit une évolution à peu près parallèle à celle de l'urée.

- La courbe d'excrétion des mucoprotéines urinaires montre une diminution presque constante au fur et à mesure de la prolongation du séjour souterrain. On note cependant dans certains cas, un maximum de 48 ou 72 heures après le début de l'expérience. Ce fait a été souligné par certains auteurs.

L'étude des moyennes des températures rectales montre une évolution très particulière : les températures, particulièrement basses au début de l'expérience, augmentent progressivement, les chiffres du matin accusant cependant assez peu de différence.

- L'analyse des estimations des durées brèves montre un allongement général qui doit être en relation directe avec l'ambiance de milieu souterrain. On remarque que l'accroissement de l'évaluation est proportionnelle au temps passé sous terre (Saumande 1963 - Saumande, Angiboust 1965).

\section{Conclusions}

Dans le domaine biologique, sous les effets de l'uniformisation des conditions du climat souterrain et de la standardisation de l'alimentation, nous constatons, après la chute des premiers jours, une stabilisation des éliminations urinaires tant sur le plan quantitatif que sur le plan qualitatif. Le même phénomène peut être observé dans le domaine de métabolisme reflété par l'évolution de la température rectale.

L'estimation de la durée suit une évolution temporelle grossièrement parallèle à celle des autres constantes biologiques.

Il nous semble pouvoir conclure que, dans ces conditions anormales d'environnement, l'homme adopterait un comportement de vie ralentie, d'activité vitale réduite, qui pourrait être interprété comme une réaction de défense contre les agressions de l'environnement d'exception.

\section{RÉSUMÉ}

L'auteur a étudié au cours de 5 expéditions spécialement conçues, le comportement de l'homme dans le milieu très spécial des cavernes. Il expose les tests utilisés.

Les résultats obtenus permettent de dire que l'organisme humain paraît s'adapter à cet environnement inhabituel, mais en adoptant un comportement de vie ralentie, d'activité vitale réduite.

\section{ABSTRACT}

In the course of live specially planned expeditions, the author studied the behavior of man in the very special environment of caves. He explains the tests employed. 
The results obtained suggest that the human organism seems able to adapt to this uncommon environment but exhibits lethargy and reduced activity.

\section{B I B LI OGRAP H I E}

Angiboust, R. (1962) - L'estimation des durées brèves, reflet de l'état d'éveil général de l'organisme, 8 p. Cerma, Paris.

LE Bideau, G. (1961) - Bilan azote et potassium en expérimentation écologique. Rapport technique P.R.Z. 21/61, 60 p. Prohuza, Paris.

- Travail et alimentation en haute altitude. In: Documentation du Cedus, Paris.

Genesseaux, P. (1956) - L'alimentation d'une troupe en climat très froid. Thèse médecine, $55 \mathrm{p}$.

Medical Nutrition Laboratory - Denver (Colorado) (1956) - Calorie intake and energy expendenture in a sub-artic environment. H.Q.R. et D.C. U.S. Army. March 1956.

Metz, B., Lambert, C., Hasselman, M., et Van Hove, R. (1959) - Métabolisme hydrominéral et bilan calorique du travailleur de force en zone saharienne. In: Etude documentaire effectuée pour le Centre d'Etude et d'Information des problèmes humains dans les zones arides, I. vol., 109 p. Paris.

Perdriel, G. (1962) - Troubles visuels observés à la suite d'un séjour prolongé dans l'obscurité. Société Médicale Française d'Education Physique et de Sport. Séance du 15. XII. 1962.

- (1962) - Le test de Farnsworth 100 Hue. Annales d'oculistique, t. CLXXXXV, No 2-1962, p. 120-130.

Placidi, Th., et Postic, F. (1955) - Ration de survie pour équipage en détresse. Médecine Aéronautique ( $2^{\mathrm{e}}$ trimestre 1955).

Rivolier, J. (1959) - Froid et altitude dans leurs rapports avec l'alimentation. Annales de la nutrition et de l'alimentation, vol. IX, No 3 .

SAUMANDE, P. (1963) - Un aspect psycho-physiologique du comportement de l'homme dans un environnement d'exception: le milieu souterrain. Etude expérimentale. Spélunca Mémoire, No 3, p. 224-231.

- (1964) - Contribution à l'étude du comportement de l'homme en milieu souterrain. Spélunca Mémoire, No 1, p. 24-26. Mars 1964.

- (1966) - Physiologie en Spéléologie. VIIe Congrès National de Spéléologie, Bordeaux (à paraître).

- (1966) - Etude de quelques facteurs physiques pouvant avoir une influence sur le comportement de l'homme séjournant dans les cavités naturelles. VII e Congrès National de Spéléologie, Bordeaux (à paraître).

Saumande, P., et Angiboust, R. (1965) - Expédition spéléologique à la cavité des Vitarelles en août 1964. Résultats biologiques et psychophysiologiques. Société de Physiologie et de Médecine Aéronautique et Cosmonautique. Séance du 19. XI. 1965.

Saumande, P., et Le Bideau, G. (1962) - Possibilité d'expérimentation physiologique sur l'homme dans un environnement d'exception: le milieu souterrain. Spélunca Mémoire, No 2, p. 132-138.

TAyeau, F. (1960) - Une manifestation de la fatigue: l'augmentation des mucoprotéines urinaires, p. 253-257. Revue Médicale Française, Juin 1960. 

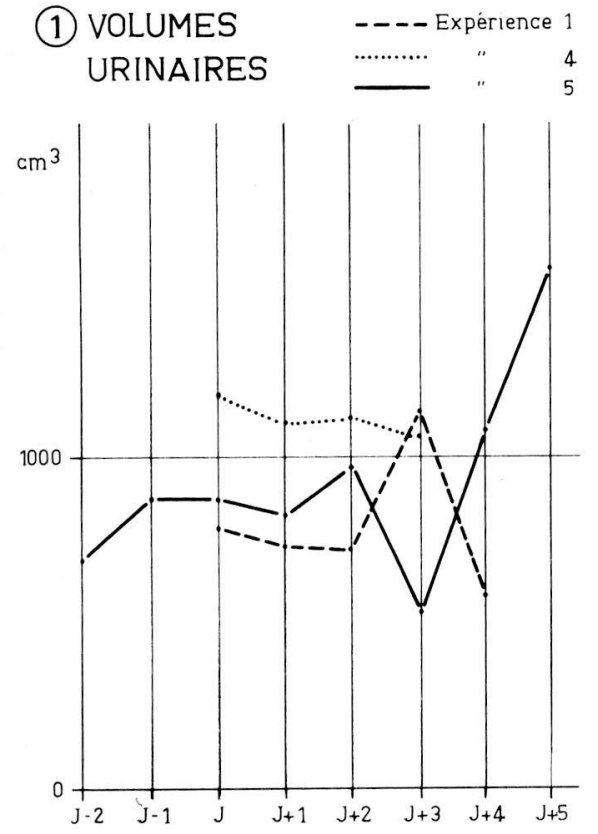

(3) TEMPERATURES RECTALES ……. Expérience 4

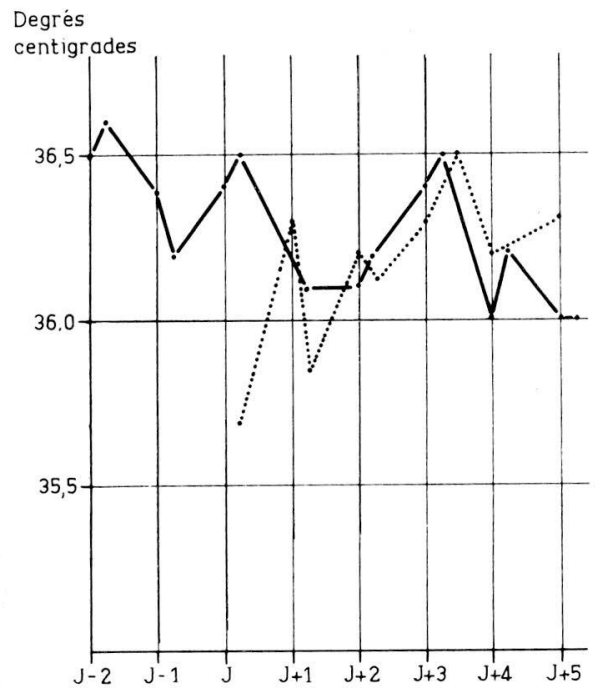

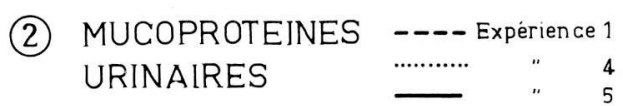

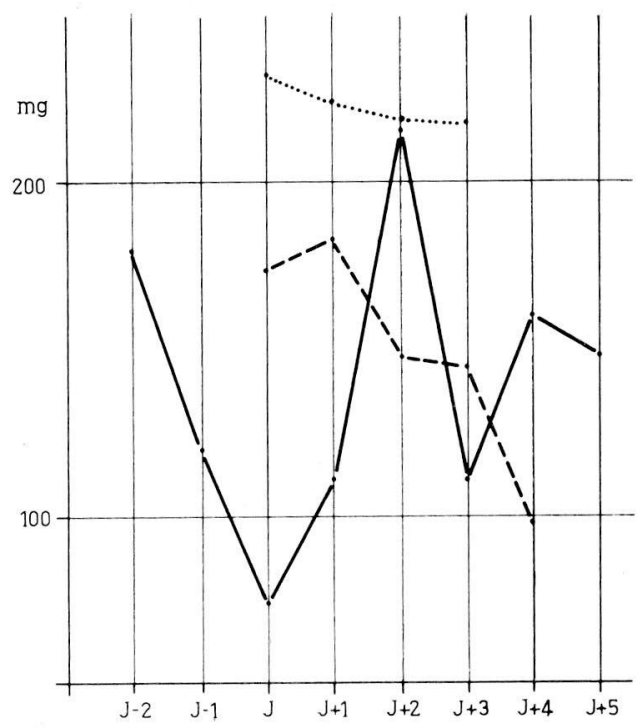

\begin{tabular}{l} 
(4) TEMPERATURES RECTALES \\
DU MATIN \\
…….... Exp. \\
\hline
\end{tabular}

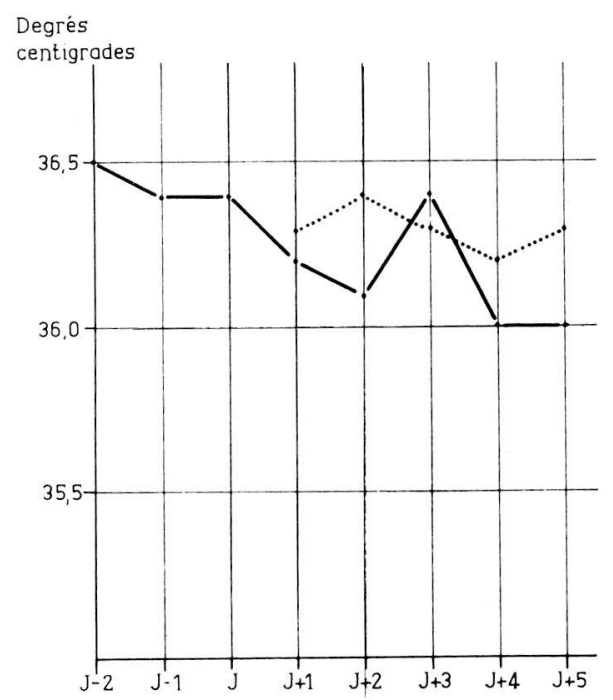


(5) TEMPERATURES

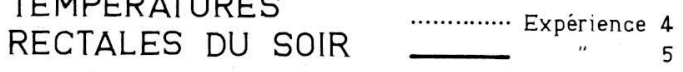
Degrés
centigrades

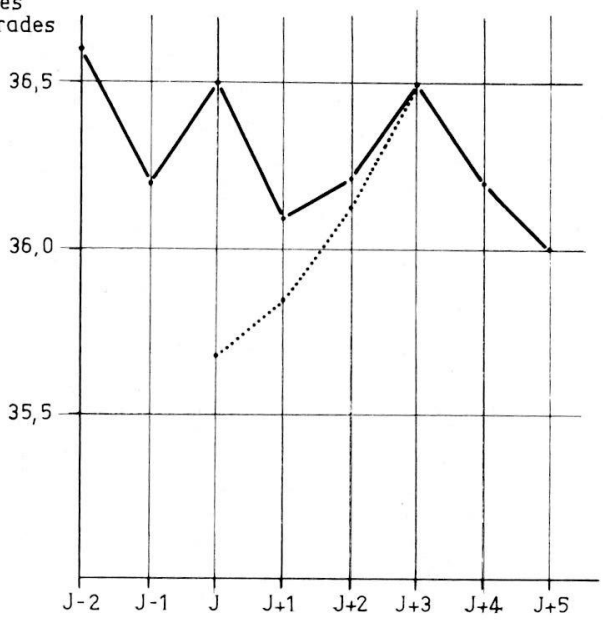

(6)

6) Estimation DURÉES BRÈVES

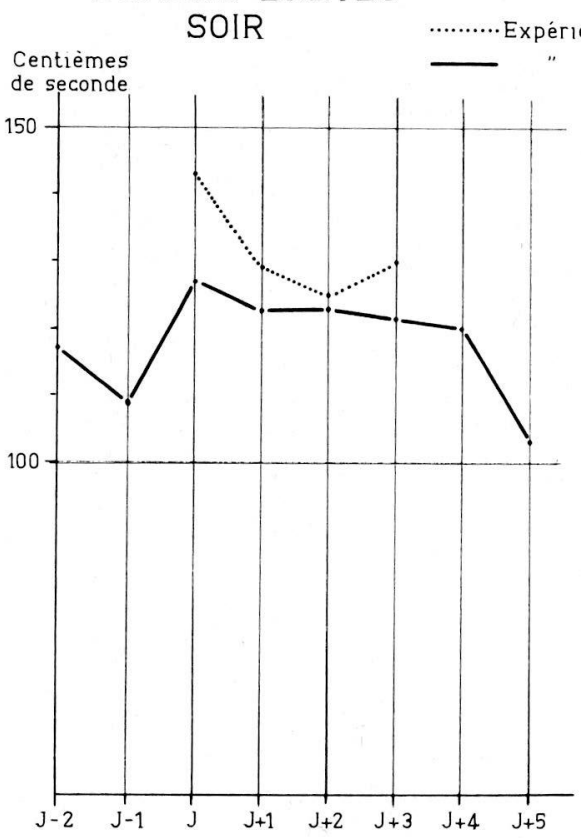

(7) ESTIMATION

\section{DURÉES BRĖVES}

MATIN

5 Centiemes de seconde

---Expérience 1
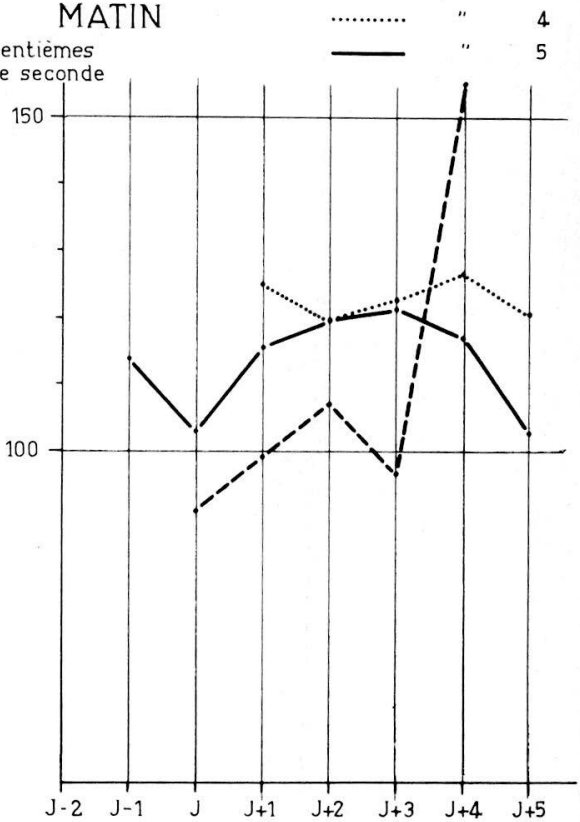